\section{COLEOPTERA (INSECTA) FAUNA FROM THE INDIAN THAR DESERT, RAJASTHAN}

\author{
Sarfrazul Islam Kazmi ${ }^{1}$ and V. V. Ramamurthy ${ }^{2}$ \\ 1 Zoological Survey of India, Desert Regional Station, Jhalamand, \\ Pali Road, Jodhpur, Rajasthan 342005, India. \\ 2 Division of Entomology, Indian Agriculture Research Institute, \\ Pusa, New Delhi 110012, India. \\ Email: ${ }^{1}$ kazmisi@rediffmail.com
}

The order Coleoptera includes 3,50,000 species, amongst which, about 1,50,88 species are known from Indian region. Roonwal (1983) and Ghosh et al. (1996) provided significant information on geographical distribution of insect species in the Thar Desert. Saha (1979) gave an excellent review on the taxonomy and distribution of the Indian blister beetles (Meloidea) and also included a few species from his study in the Thar Desert. Tak and Sewak (1987) and Tak (1996) reported 27 species of aquatic beetles from Jodhpur and Sikar districts belonging to families Haliplidae, Gyrinidae, Dytiscidae and Hydrophilidae which have been included to prepare this checklist. Tandon (1996) reported 34 species, and identified gaps in research on the distribution of coleopteran species from the Thar Desert of Rajasthan.

In view of filling the gaps in our knowledge on the fauna of Thar Desert, Rajasthan, the present study was initiated under a research project entitled "Studies on faunal diversity in the Thar desert of Rajasthan" sanctioned by the Ministry of Environment and Forests, New Delhi, at Zoological Survey of India, Desert Regional Station, Jodhpur. In this paper we have reported 99 species belonging to 60 genera under 13 families of Coleoptera from Thar desert of Rajasthan during May, 2000June 2003. Besides, eight specimens have been identified up to generic level.

Majority of the specimens were collected during the day from agricultural as well as barren lands or sand dune areas by the free sweeping method. However, some groups of insects were collected during night with the help of screen light trap with a strong source of white light (Sengupta \& Mukhopathyay, 1990). All the specimens are deposited in the National Zoological Collection, Desert Regional Station, Zoological Survey of India, Jodhpur. This checklist covers all the districts of the Thar Desert. In Ganganagar, Hanumangarh, part of Bikaner and Jaisalmer the crop pattern has entirely changed during the last two decades due to the Indira Gandhi canal.

\section{REFERENCES}

Ghosh, A.K., Q.H. Baqri and I. Prakash (1996). Faunal diversity in the Thar Desert: Gaps in Research. Scientific Publication, Jodhpur i-xi, 1140 .

Roonwal, M.L. (1983). Fauna of Great Indian Desert. In: Desert Resource and Technology. Scientific Publication, Jodhpur 1:1-85.

Saha, G.N. (1979). Revision of Indian Blister beetles (Coleoptera: Meloidea: Meloinae). Records of the Zoological Survey of India 74(1): 1146.

Sengupta, T. and P. Mukhopathyay (1990). Coleoptera, pp.151-157.
Table 1. Coleoptera from Thar Desert of Rajasthan

\begin{tabular}{ll}
\hline Scientific name & Localities \\
\hline Meloidae & \\
Mylabris phalerata (Pallas) & Barmer: Bithuja (CV) \\
Mylabris pustulata Thunberg & Barmer: Bithuja (CV), \\
& Jodhpur: Rohit (CV) \\
Cylindrothorax audoniui (Haag-Rutenberg) & Barmer: Pachpadra (CV) \\
Cyaneolytta coerulea (Leuckart) & Barmer: Ashotra (CV) \\
Cyaneolytta pictus (Laporte) & Jodhpur: Bhopalgarh (CV), \\
& Jaisalmer: Chandan, Sam (CV) \\
Cyaneolytta violacea Brandt & Barmer: Ashotra (CV) \\
Pseudosybaris kempi Saha & Bikaner (CV)
\end{tabular}

Cicindelidae

Cicindela cancellata Dej.

Cicindela minuta Oliver

Cicindela sumatrensis var imperfecta

W. Horn.

Jaisalmer: Chandan (CV), Jalore (CV) Jodhpur: Agolai (Ar), Bikaner: Mukham (Ar)

Jaisalmer: Chandan (CV)

\section{Carabidae}

Carabus orientalis Hope

Anthia sexmaculata (Fab.)

Calosoma maderae Fab.

Bikaner (CV)

Jodhpur: Dechu (Ar)

Bikaner (CV)

\section{Hydrophilidae}

Hydrous senegalensis Perch

Hydrous olivaceus Fab.

Hydrous indicus Bdel.*

Helochares crematus Regimbart*

Helochares lentus Sharp*

Enochrus flavicans Regimbart*

Coleostoma horni Regimbart*

Coleostoma stuttum Walker

Sternolopus rufipes Fab.*

Berosus nigriceps (Fab.)

Berosus pulchellus Mcleay

Berosus indicus Mots*

Berosus fairmairizait

Jaisalmer: Chandan (WB)

Jodhpur: Kailana Lake (WB)

Jodhpur: Kailana Lake (WB)

Jodhpur: Kailana Lake (WB)

Jodhpur: Kailana Lake (WB)

Jodhpur: Kailana Lake (WB)

Jodhpur: Kailana Lake (WB)

Jodhpur: Kailana Lake (WB)

Jodhpur: Kailana Lake (WB),

Bikaner (WB)

Sikar (WB)

Sikar (WB)

Jodhpur: Kailana Lake (WB)

Sikar (WB)

\section{Haliplidae}

Halipus (Liaplus) pulchellus indicus

Regimbart*

Jodhpur: Kailana Lake (WB)

\section{Gyrinidae}

Orectochilus (Patrus) limbatus Regimbart* Jodhpur: Kailana Lake (WB)

\section{Dytiscidae}

Guignotus pendjabensis Guignot*

Guignotus flammulatus (Reigmbart)*

Herophydrus musicus Klug.*

Hyphoporus kempi Gschwendtner*

Hyphoporus severini Regimbart"

Hyphoporus flavicans Regimbart

Laccophilus flexuosus Aube*

Laccophilus sharpi Regimbart*

Laccophilus parvulus Aube ${ }^{\#}$

Laccophilus chinensis inefficiens Walker\#

Canthydrus laetabilis (Walker)*

Erectes sticticus Linn.*

Hydaticus fabricii Macleay*

Hydaticus vittatus Fab.*

Hydaticus histrio Clark

Cybister (Meganectes) tripunctatus

asiaticus Sharp*

Rhantus congestus (Klug)\#

Jodhpur: Kailana Lake (WB) Jodhpur: Kailana Lake (WB) Jodhpur: Kailana Lake (WB) Jodhpur: Kailana Lake (WB) Nagaur: Nawa, Guda (WB) Jodhpur: Kailana Lake(WB), Sikar (WB)

Jodhpur: Kailana Lake (WB) Jodhpur: Kailana Lake (WB) Nagaur: Nawa, Guda (WB), Sikar (WB)

Nagaur: Nawa (WB) Jodhpur: Kailana Lake (WB) Jodhpur: Kailana Lake (WB), Sikar (WB)

Jodhpur: Kailana Lake (WB) Jodhpur: Kailana Lake (WB) Bikaner (WB) Jodhpur: Kailana Lake (WB), Sikar (WB)

Jodhpur: Kailana Lake (WB)

\section{Coccinellidae}

Coccinella septempunctata Linnaeus

Jodhpur: Osian, Piparcity (CV), Phallodi (CV), Pali: Sumerpur(CV) Ganganagar(CV).

Coccinella septempunctata var divaricata Churu: Talchapper (CV)

Cycloneda carolinae Muls. Jaisalmer (CV)

Thea bisoctonota Muls. 


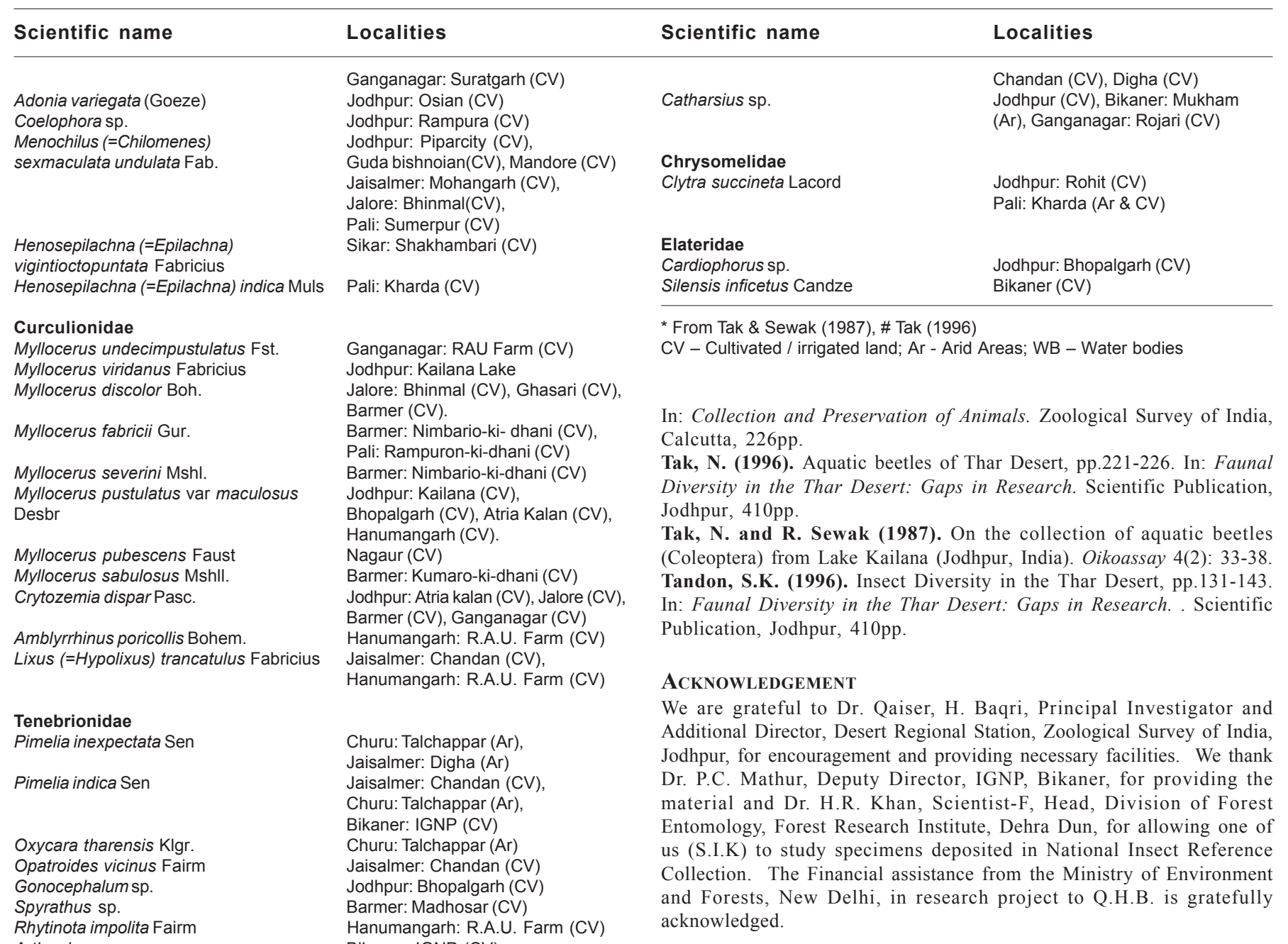

Rhytinota impolita Fairm

Arthrodes sp.

Setenis semiopaca Stebbing

Bikaner: IGNP

Jodhpur: Osian (CV)

Bikaner (CV)

Scarabaeidae

Scarabaeus cristatus Fabricius

Scarabaeus gangeticus Cast.

Scarabaeus brahminus Cestlenau

Barmer: Madhosar (CV)

Bikaner: R.A.U. Farm (CV)

Bikaner (CV)

Barmer: Akoli (Ar), Jaisalmer (Ar)

Bikaner (CV)

Onthophagus catta Fabricius

Onthophagus bonasus Fab.

Jodhpur (CV), Jaisalmer: Chandan

(CV), Bikaner: R.A.U. Farm (CV)

Onthophagus sp.

Gymnopleurus cyaneus (Fabricius)

Hybosorus orientalis (Westwood)

Bikaner: R.A.U. Farm (CV)

Jodhpur: Kailana lake (CV)

Jodhpur: Bhopalgarh (CV)

Jaisalmer: Chandan (CV)

Jodhpur: Bhopalgarh (CV)

Jodhpur: Bhopalgarh (CV),

Pali: Sumerpur (CV),

Jaisalmer: Sam (Ar)

Bikaner (CV)

Bikaner (CV)

Bikaner (CV)

Bikaner (CV)

Jaisalmer: Sam (Ar), Jalore:

Ahore (CV), Jodhpur: Mathania

(CV), Bikaner: Mukham (CV)

Jaisalmer: Chandan (CV)

Jodhpur: Bhopalgarh(CV,

Pali: Sanderao (Ar \& CV),

Jaisalmer: Dabla (Ar).

Jodhpur: Mathania (CV)

Autoserica insanabilis Brsk.

Hanumangarh (CV), Jaisalmer:

Catharsius pithecus Fab. 